\title{
Leukocytoclastic Vasculitis as a Cutaneous Manifestation of COVID-19 Infection with a Positive Skin Antigen Test
}

\author{
Oghowan Abdelrahman $^{\mathrm{a}} \quad$ Andleeb Shadan $^{\mathrm{b}} \quad$ Laila Al Dabal $^{\mathrm{b}}$ Tasnim R. Keloth $^{\mathrm{c}}$ \\ aCardiology Department, Rashid Hospital, Dubai Health Authority, Dubai, United Arab Emirates; 'bnfectious Disease \\ Department, Rashid Hospital, Dubai Health Authority, Dubai, United Arab Emirates; ${ }^{~}$ Histopathology Department, \\ Dubai Hospital, Dubai Health Authority, Dubai, United Arab Emirates
}

\section{Keywords}

COVID-19 · Immunology · Infectious · Treatment · Vasculitis

\begin{abstract}
On 11 March 2020, WHO declared the SARS-CoV-2 outbreak as a pandemic, representing a public health emergency of unprecedented dimension. COVID-19 is a multisystem Novel viral infection with protean manifestations and variable degrees of clinical presentation. The most common presentation is upper and lower respiratory tract illness. Cutaneous manifestations of COVID-19 have rarely been described. In this case report, we present a 41-year-old male patient who presented with fatigue and follicular skin rash followed almost 2 weeks later with fever, left upper limb transient weakness, and pneumonia. Of interest is that the patient's skin lesions tested positive on COVID-19 antigen test. The patient received a course of anti-COVID-19 treatment as per our national guideline, and he made a full clinical and radiology recovery.

(c) 2021 The Author(s)

Published by S. Karger AG, Basel
\end{abstract}

karger@karger.com www.karger.com/dmj

Karger $\stackrel{\text { ' }}{5}$

GOPEN ACCESS
(C) 2021 The Author(s).

Published by S. Karger AG, Basel

This article is licensed under the Creative Commons AttributionNonCommercial-NoDerivatives 4.0 International License (CC BYNC-ND) (http://www.karger.com/Services/OpenAccessLicense) Usage and distribution for commercial purposes as well as any distribution of modified material requires written permission.

\section{Introduction}

This study suggests that skin manifestations could be an early presentation of COVID-19 and during outbreaks; they should be taken into proper account to enhance the crucial activity of case finding and early case isolation. To our knowledge, this is the first reported case of COVID-19 infection with positive skin antigen test, and it has been diagnosed and managed entirely in our hospital setting. Further studies in larger cohorts of patients are needed to better understand the several aspects of the cutaneous involvement of COVID-19 and its association with the disease infectivity, course, and severity.

\section{Case Presentation}

On April 16, 2020, a 41-year-old male who is known to be diabetic, presented to our emergency with fatigue and multiple, nontender pruritic lower limb folliculocentric papules. It progressed slowly to involve his lower back, arms, and fingers, sparing only his face, scalp, abdomen, and genitalia. Almost 2 weeks later, the patient developed fever, cough, and sudden left upper limb weakness which made him seek medical advice. Clinically, he looked well with stable vital signs. Dermatological examination showed confluent macules, papules, and petechiae in both legs, extending 

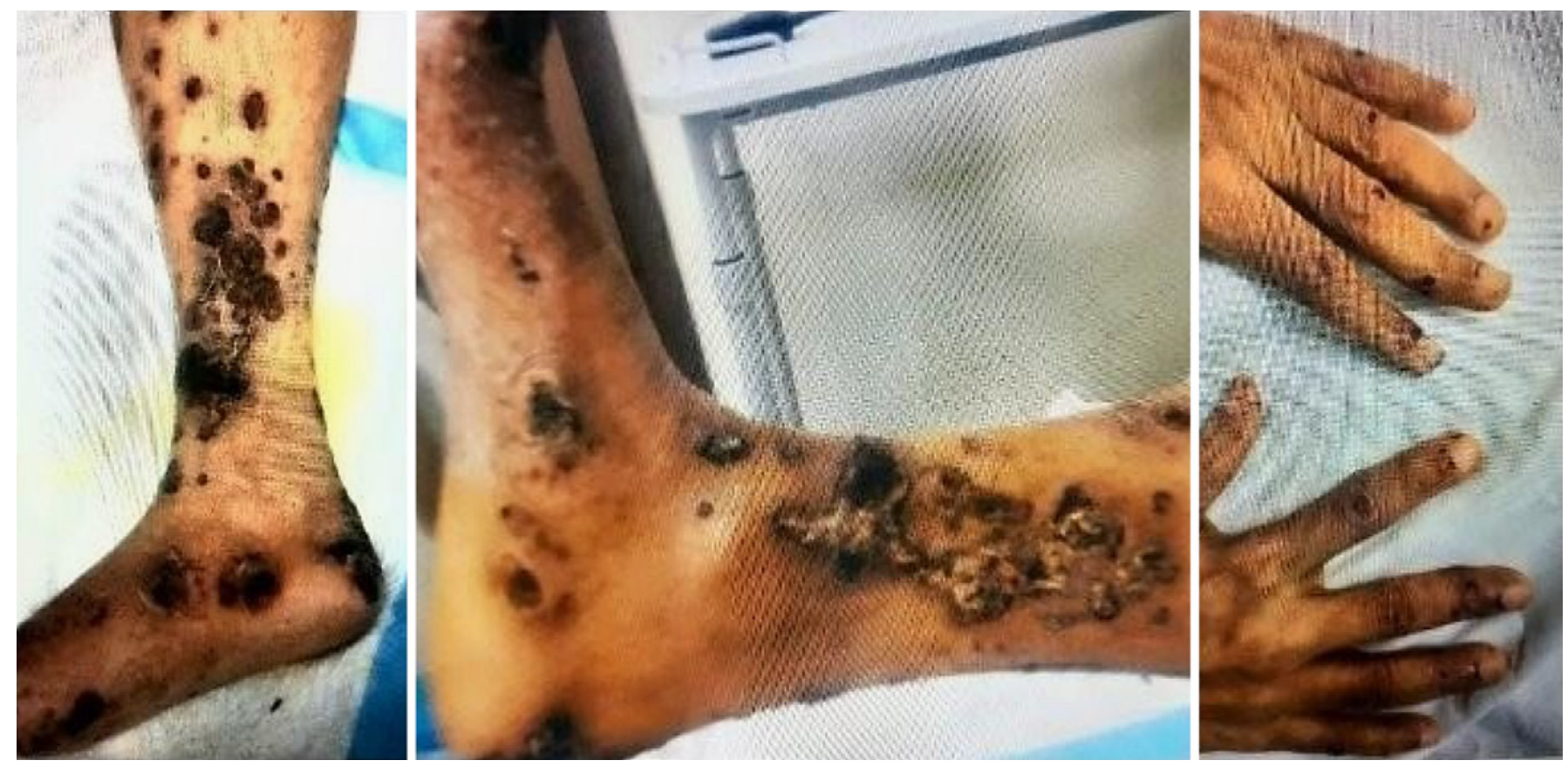

Fig. 1. Macroscopic view of the lesions over the anterior and medial aspect of the lower limbs and the fingers. The fingers showed acral (ischemic hemorrhagic) dots, and small papules.

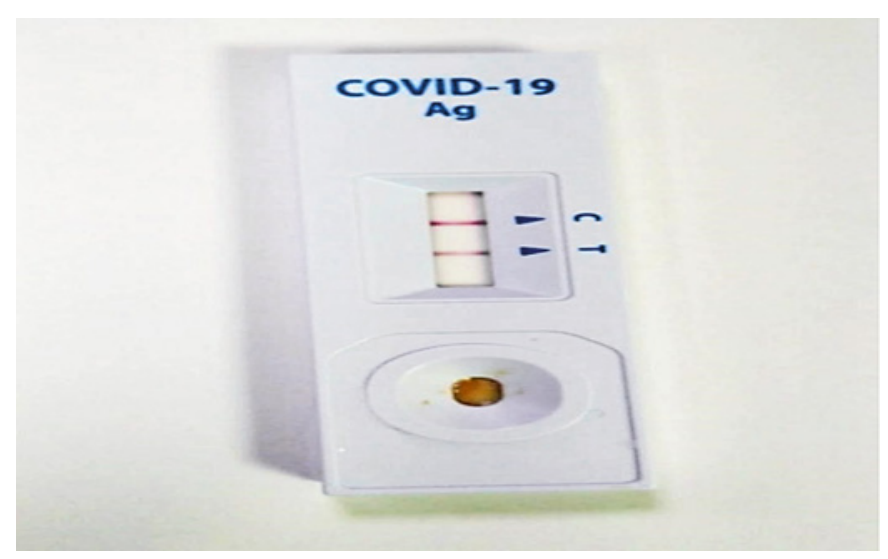

Fig. 2. COVID-19 skin antigen test $=$ positive .

from the ankles up to the thighs and lower back. A striking absence of lesions in the crural folds was noted. His palmar and plantar surfaces were involved. Some macules showed ulceration with black sloughed necrotic skin. No mucosal lesions, no nodules, livedo reticularis, and nor retiform purpura were found. His fingers showed features of Acral (ischemic hemorrhagic), dots, and small papules (Fig. 1).

Investigations showed poor controlled diabetes with HBA1c at $13.2 \%$. He had normal hemoglobin and platelet levels with high WBCs at $26 \times 10^{3} / \mu \mathrm{L}$ (neutrophil predominant with absolute lymphocyte count at $\left.1 \times 10^{3} / \mu \mathrm{L}\right)$. His C-reactive protein was high at
$305.5 \mathrm{mg} / \mathrm{L}(0-5 \mathrm{mg} / \mathrm{L})$ and procalcitonin at $0.2 \mathrm{ng} / \mathrm{mL}$. Urine was devoid of protein or blood.

Ferritin was high at $1,387 \mathrm{ng} / \mathrm{mL}, \mathrm{LDH}$ at $418 \mathrm{U} / \mathrm{L}$, and Dimer at $9.2 \mu \mathrm{g} / \mathrm{mL}$. Serology for Hepatitis B, Hepatitis C, and HIV was negative. Antinuclear factor, anti-double strand DNA, antinuclear cytoplasmic antibody, extractable nuclear antigen panel, rheumatoid factor, and cryoglobulins were negative. Nasopharyngeal swab for SARS-Cov-2 was positive on reverse transcription PCR. Baseline chest X-ray and CT brain were reported as normal.

The skin rash was proven to be COVID-19 related as the skin antigen test done by the fluid obtained from the rash was positive (Fig. 2). Three 4-mm punch biopsies were taken for histopathological examination, from the lesions in the anterior upper right thigh. The histopathological examination showed partial to fullthickness epidermal ulceration covered by serum crusts with dense aggregates of neutrophils, and an intraepidermal vesicle containing abundant neutrophils and some eosinophils. The underlying dermis revealed features of vasculitis involving superficial and mid-dermal small to medium-sized blood vessels, showing neutrophilic infiltrate with leukocytoclastic and nuclear dust, RBC extravasation, focal mild fibrin extravasation, and dense perivascular and focal peri adnexal neutrophilic inflammatory infiltrate. No fungal organism was identified. The diagnosis of leukocytoclastic (hypersensitivity) vasculitis was confirmed on histologic examination, and it was proven to be COVID-19 related as his skin antigen testing obtained from the rash was strongly positive as well (Fig. 3).

From the day of admission, he was started on oral hydroxychloroquine therapy (based on national guideline) as well as intravenous antibiotics, therapeutic doses of anticoagulation with lowmolecular-weight heparin and low-dose aspirin. Seventy-two 

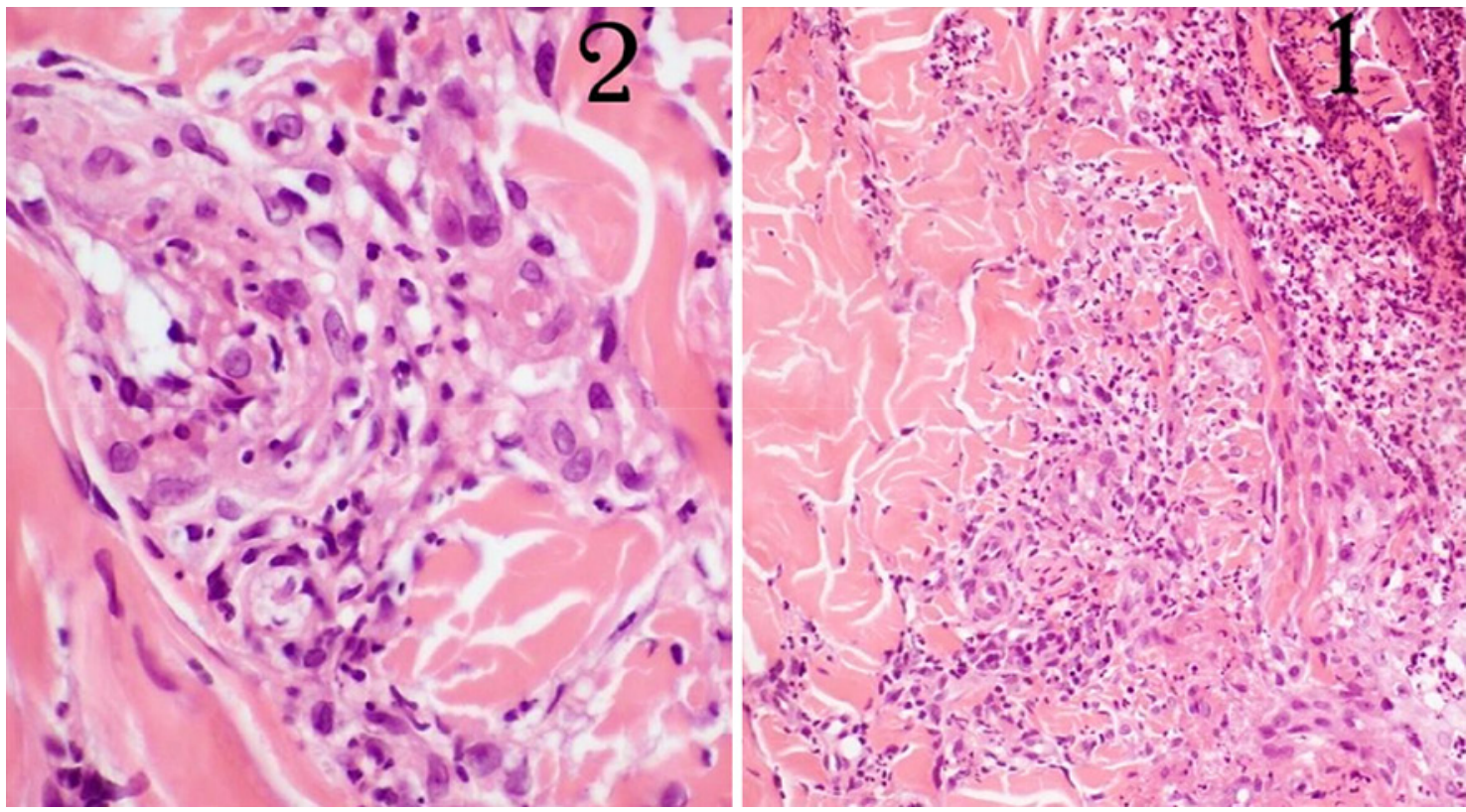

Fig. 3. Histopathology: Slide 1 Ulcerated area covered by abundant serofibrinous necrotic material, an intraepidermal neutrophilic blister, and busy dermis showing leukocytoclastic vasculitis with neutrophilic infiltrate in a perivascular and interstitial pattern. Slide 2 Showing blood vessel wall being infiltrated by neutrophils and the presence of karyorrhectic nuclear dust.
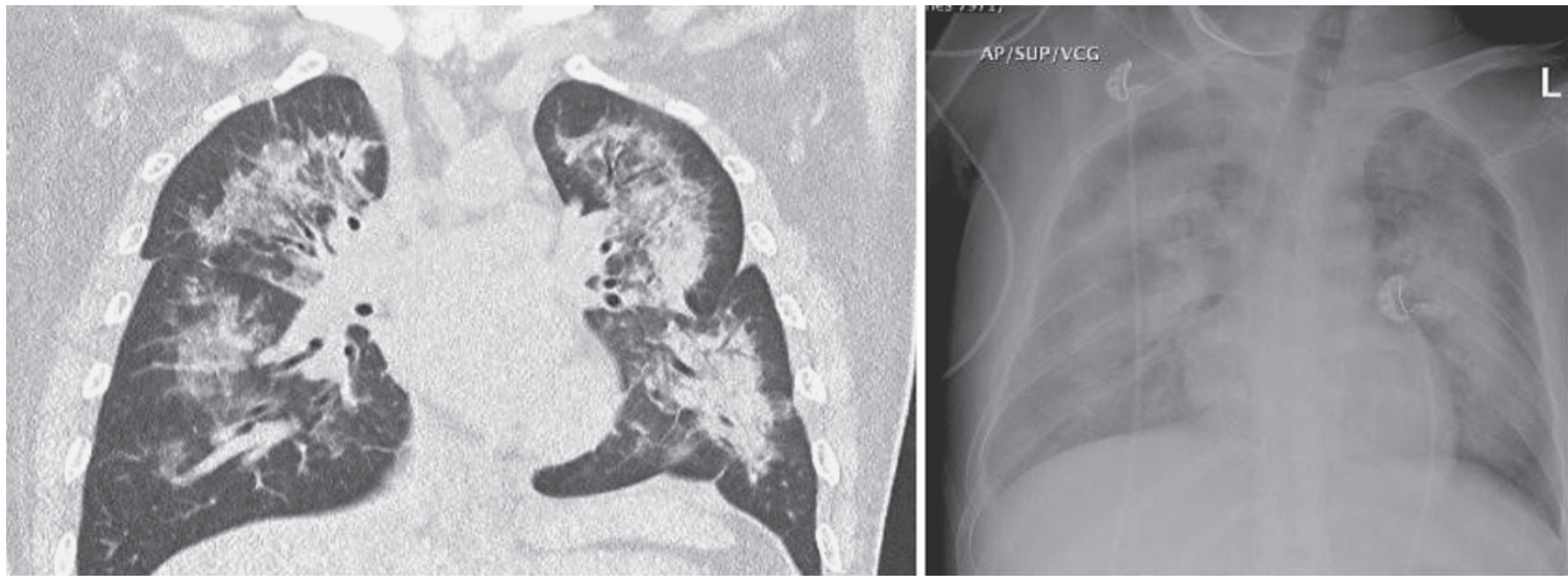

Fig. 4. Chest X-ray and CT Chest (72 h post-hospitalization): showed bilateral, widespread, consolidative pattern with air bronchogram involving both lung fields and background ground-glass opacity. Moderate bilateral pleural effusion, more evident on the right side.

hours post-hospitalization, he complained of increasing cough, shortness of breath, and his oxygen requirement increased to highflow nasal cannula. A chest X-ray and computed tomography chest radiograms both done and showed consolidative pattern with air bronchogram involving both lung fields with scattered patchy areas of ground-glass opacities, normal heart size with no pericar- dial effusion, no pneumothorax, or evidence of pulmonary embolism (Fig. 4). Echocardiography showed evidence of cardiomyopathy with markedly impaired global LV systolic function with estimated EF of 30-35\%. Following the development of severe pneumonia, his anti-COVID-19 treatment regimen was escalated with the addition of lopinavir-ritonavir and favipiravir. The pa- 

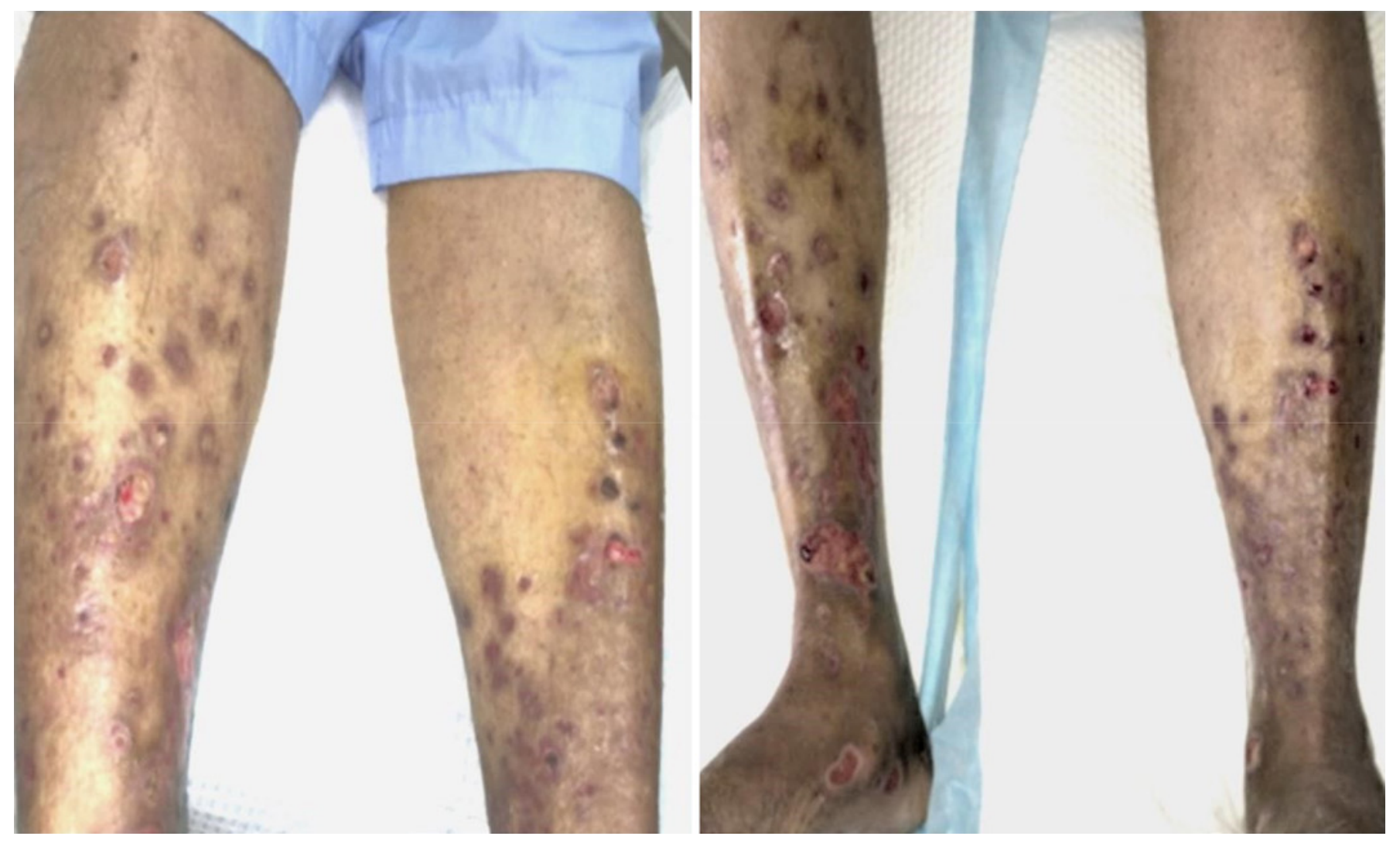

Fig. 5. Macroscopic view - post-treatment: showed resolution and healing of the main skin lesions.

tient's respiratory status worsened with the progression of pneumonia, justifying the addition of tocilizumab as per our national treatment guidelines, in addition to optimizing oxygen requirements, prone positioning, and full anticoagulation.

During his hospital stay, the patient did not require intubation or mechanical ventilation. He made an excellent clinical and radiological recovery over 4 weeks period, and he was discharged home with oxygen saturation of $100 \%$ on room air and fully resolved cutaneous lesions (except for few residual areas of hypo and hyperpigmentation) (Fig. 5).

\section{Discussion/Conclusion}

On 11 March 2020, WHO declared the SARS-CoV-2 outbreak as a pandemic, representing a public health emergency of unprecedented dimension [1]. Cutaneous manifestations of COVID-19 are not very common, and most published data is in the form of case series and few case reports. The mechanisms of cutaneous manifestations of COVID-19 are so far not well-elucidated. A clear understanding as to whether cutaneous mani- festations are secondary consequences of lung-related infections or primary infection of the skin is lacking. The largest group was published by Recalcati (2020) who reported 88 patients positive for COVID-19 and out of the 18 had cutaneous manifestations accounting to $20.4 \%$ of total cases. Erythematous rash seemed to be the most prominent pattern (14/18 patients- $77.8 \%)$, followed by an urticarial rash (3/18 - 5.6\%). Vesicular rash was seen in 1 patient (5.6\%) [2]. Galván Casas et al. [3] described 5 patterns of skin manifestations in a total of 375 patients with COVID-19. The most common pattern was of pseudo chill blains $19 \%$ with acral areas of involvement. Vesicular eruptions were seen in 9\%, urticarial and maculopapular rash in 19 and 47\%, respectively, and livedo reticularis/necrosis was seen in $6 \%$ of cases involving truncal and acral areas. This study also looked into the demographics, the temporal relation at presentation, and other clinical manifestations [3]. Livedo reticularis/necrotic lesions were associated with severe disease and seen in elderly patients with high mortality. 
Theories suggest that the viral particles present in the cutaneous blood vessels in patients with COVID-19 infection could lead to a lymphocytic vasculitis similar to those observed in thrombophilia arteritis induced by blood immune complexes that activate cytokines [4]. Acral, ischemic hemorrhagic macules with cutaneous necrosis, was the pattern of rash observed in our patient and can seemingly be explained by most of the abovementioned mechanisms. The microscopic pattern of leukocytoclastic reaction similar to the one described in our patient has also been reported by Jimenez-Cauhe et al. [5] who described as mild to moderate spongiosis in the epidermis and dilated vessels In dermis filled with neutrophils, extravasated RBCs, lymphocytic perivascular, and interstitial infiltrate. It is still unclear whether cutaneous symptoms are a secondary consequence of respiratoryrelated infection or primary infection of the skin itself. It is more likely that a combination of such mechanisms is responsible for the cutaneous manifestations found in COVID-19+ individuals [6-8]. To our knowledge, this is the first reported case of COVID-19 infection with a positive skin antigen test which gives direct evidence of cutaneous viral invasion. This case highlights the multifaceted presentation of COVID-19 infection and that physicians in different subspecialties need to be aware of these uncommon manifestations.

\section{Statement of Ethics}

Written informed consent was obtained from the patient for publication of this case report and any accompanying images.

\section{Conflict of Interest Statement}

The authors have no conflicts of interest to declare.

\section{Funding Sources}

No funding or sponsorship was received for this study or publication of this article.

\section{Author Contributions}

The authors have equally contributed to collecting data and preparation of the manuscript.

\section{References}

1 World Health Organization. WHO coronavirus disease (COVID-19) dashboard. WHO website. Accessed November 25, 2020. Available at WHO Coronavirus Disease (COVID-19) Dashboard|WHO Coronavirus Disease (COVID-19) Dashboard.

2 Hedou M, Carsuzaa F, Chary E, Hainaut E, Cazenave-Roblot F, Masson Regnault M. Comment on "Cutaneous manifestations in COVID-19: a first perspective" by Recalcati S. J Eur Acad Dermatol Venereol. 2020;34(7): e299-300.

3 Galván Casas C, Català A, Carretero Hernández G, Rodríguez-Jiménez P, Fernández-Nieto D, Rodríguez-Villa Lario A, et al. Classifi- cation of the cutaneous manifestations of COVID-19: a rapid prospective nationwide consensus study in Spain with 375 cases. Br J Dermatol. 2020;183(1):71-7.

4 Papa A, Salzano AM, Di Dato MT, Varrassi G. Images in practice: painful cutaneous vasculitis in a SARS-Cov-2 IgG-positive child. Pain Ther. 2020;9(2):805-7. .

5 Jimenez-Cauhe J, Ortega-Quijano D, Carretero-Barrio I, Suarez-Valle A, Saceda-Corralo D, Moreno-Garcia Del Real C, et al. Erythema multiforme-like eruption in patients with COVID-19 infection: clinical and histological findings. Clin Exp Dermatol. 2020;45(7):8925.
6 Sachdeva M, Gianotti R, Shah M, Bradanini L Tosi D, Veraldi S, et al. Cutaneous manifestations of COVID-19: report of three cases and a review of literature. J Dermatol Sci. 2020;98(2):75-81.

7 Gianotti R, Zerbi P, Dodiuk-Gad RP. Clinical and histopathological study of skin dermatoses in patients affected by COVID-19 infection in the Northern part of Italy. J Dermatol Sci. 2020;98(2):141-3.

8 Guo YR, Cao QD, Hong ZS, Tan Y-Y, Chen $\mathrm{S}-\mathrm{D}$, Jin $\mathrm{H}-\mathrm{J}$, et al. The origin, transmission and clinical therapies on coronavirus disease 2019 (COVID-19) outbreak: an update on the status. Mil Med Res. 2020;7(1):11. Published 2020 Mar 13 